\title{
Spatial measurement of spurious forces with optical tweezers
}

Ignacio Bordeu Giovanni Volpe Juan Pablo Staforelli 


\title{
Spatial measurement of spurious forces with optical tweezers
}

\author{
Ignacio Bordeu ${ }^{a}$, Giovanni Volpe ${ }^{b}$ an Juan Pablo Staforelli ${ }^{a}$ \\ ${ }^{a}$ Center for Optics and Photonics, Universidad de Concepción, Casilla 4016, Concepción, Chile \\ ${ }^{c}$ Department of Physics, Bilkent University, Cankaya, Ankara 06800, Turkey
}

\begin{abstract}
The study of diffusion in a crowded and complex environment, such as inside a cell or within a porous medium, is of fundamental importance for science and technology. Combining blinking holographic optical tweezers and sub-pixel video microscopy permits one to study Brownian motion in confined geometries. In this work, in particular, we have studied the Brownian motion of two colloidal particles interacting hydrodynamically with each other. The proximity between the two microspheres induces a space-dependence in the particles diffusion coefficient and, therefore, a spurious drift. We measure this drift and evaluate the magnitude of the spurious force associated with it. We present the optoelectronic tools employed in the experiment and we discuss the experimental results.
\end{abstract}

Keywords: Brownian motion, stochastic differential equations, diffusion gradients, holographic optical tweezers, blinking optical tweezers, digital video microscopy.

\section{INTRODUCTION}

Diffusion gradients emerge naturally when a Brownian particle is diffusing in a complex or crowded environment. For example, the bulk diffusion coefficient decreases when a particle is close to a wall due to hydrodynamic interactions, ${ }^{1}$ where the diffusion coefficient increases with the particle-wall distance approaching the corresponding bulk value at a distance of several particle radii away from the wall.

Currently, refined experiments have settled novel perspectives against the standard interpretation of Brownian motion which standardize random and unpredictable trajectories. For example, it was found that Brownian trajectories involve hydrodynamic memory, i.e. movement information is stored in the displaced fluid of the particle motion, so that the latter is not completely random but biased by feedback. ${ }^{2}$

It has been observed how the diffusion parameter depends on the geometric confinement of the particleto-wall proximity, which generates displacement of the particles from its average position in the absence of deterministic forces, thus named spurious force. ${ }^{3,4}$ Such a spurious drift play a fundamental role not only in the thermodynamics comprehension of confined geometries with synthetic beads but in natural scenarios as well. ${ }^{3,4}$

In the experiment we combine holographic optical tweezers to place two acrylate micro-spheres immersed in water in close proximity and then sub-pixel digital video microscopy to measure their position in the absence of external forces. Analyzing these data we are able to determine both the diffusion and drift parameter from its average position in order to get new insights for modeling and control of this type of process at the micro-scale.

\section{THEORETICAL BACKGROUND}

We consider the one-dimensional, time-independent Langevin equation describing the motion of a Brownian particle in the $x$-direction:

$$
\gamma \frac{d x}{d t}+\frac{d U(x)}{d x}=\sqrt{2 D} \xi(t)
$$

where the first term correspond to the viscous drag force, $\gamma=6 \pi \eta a$ is the hydrodynamic drag coefficient for a micro-sphere of radius $a$ immersed in a fluid with viscosity $\eta$; the second term correspond to the deterministic force associated to a potential $U(x)$; and the third term is the random force, where $D=k_{B} T / m \gamma$ is the diffusion

Mail address: Casilla 160-C, Concepción, Chile

E-mail: juanpablo.staforelli@cefop.udec.cl, Telephone: +56-41-266 1339

8th Iberoamerican Optics Meeting and 11th Latin American Meeting on Optics, Lasers, and Applications,

edited by Manuel Filipe P. C. Martins Costa, Proc. of SPIE Vol. 8785, 878509

(C) 2013 SPIE $\cdot$ CCC code: $0277-786 X / 13 / \$ 18 \cdot$ doi: $10.1117 / 12.2027533$ 
parameter in bulk, $k_{B}$ is the Boltzmann constant $\left(1.38 \times 10^{-23} \mathrm{~J} / \mathrm{K}\right), T$ the room temperature in Kelvin and $\xi(t)$ is a white noise, i.e., a delta-correlated random function with zero mean. The inertial term has been neglected because a micron-sized particle trapped in water behaves as an extremely overdamped oscillator, ${ }^{5}$ where the viscous drag term involving $\gamma$ will dominate the inertial term involving the mass.

To gain an intuitive understanding of the phenomenon we want to study we will follow Lancon et al. ${ }^{6}$ In the absence of forces, e.g., when the optical trap is switched off and assuming there are no other potential forces, the second term of eq. 1 vanishes. We will consider how the particle moves in a (simplified) timestep. We replace the velocity by $\Delta x / \Delta t$ and the noise term by $1 / \Delta t$, we get

$$
x(t+\Delta t)=x(t) \pm \frac{1}{\gamma} \sqrt{2 D \Delta t},
$$

where the \pm sign is used because the Brownian walker can move either in one direction of in the other. When the diffusion coefficient became position dependent, i.e., $D=D(x)$, the length of the position-dependent step $\sqrt{2 D \Delta t}$ is not uniquely determined. In other words, it depends of the value $x(t)$ chosen along the step length, i.e., the initial point $x(t)=x$, some point in the middle or the arrival point $x(t+\Delta t)=x+\Delta x$. Thus, the diffusion coefficient is redefined as $D(x+\alpha x)$, where $\alpha=0,1 / 2$ or 1 depending of the chosen convention. With this, this coefficient can be simple expanded as

$$
D(x+\alpha x)=D(x)+\alpha \frac{d D(x)}{d x} \Delta x .
$$

Combining eq. 3 into eq. 2 gets the Brownian walker with a position-dependent diffusion coefficient

$$
x(t+\Delta t)=x(t) \pm \frac{1}{\gamma} \sqrt{2 D[x(t)] \Delta t}+\alpha \frac{d D(x)}{d x} t,
$$

A shift of the mean position distribution appear when taken statistical average on eq. 4

$$
<x(t)-x(0)>=\alpha \frac{d D(x)}{d x} \Delta t .
$$

Where the derivative with respect to the position is the so called spurious drift (see Fig. 2). The choice of $\alpha$ depends of the physics of the phenomena, e.g., if $T=1$, means the temperature is uniform and $\gamma$ is position depended.

All these considerations can be made mathematically more precise and apply to all stochastic differential equations where some multiplicative noise is present. In fact the integration of a stochastic differential equation with multiplicative noise presents some difficulties due to the irregularity of the Wiener process. In fact, the

stochastic integral $\int_{0}^{t} \sqrt{2 D(x(s))} \circ_{\alpha} d W(s) \equiv \lim _{N \rightarrow \infty} \sum_{n=0}^{N-1} \sqrt{2 D\left(x\left(t_{n}\right)\right)} \Delta W\left(t_{n}\right)$, where $t_{n}=\frac{n+\alpha}{N} T$ and $\alpha \in$ $[0,1]$, may lead to different values for different choices of $\alpha$. Common choices are: the Itô integral with $\alpha=0 ;{ }^{7}$ the Stratonovich integral with $\alpha=0.5 ;^{8}$ and the anti-Itô or isothermal integral with $\alpha=1 .{ }^{9}$ Therefore, a complete model is defined by the Langevin equation and the relative convention, ${ }^{10}$ which must be determined on the basis of the available experimental data. The convention $\alpha=1$ emerges naturally in physical systems in equilibrium with a heat bath such as a Brownian particle, as it was predicted theoretically ${ }^{11,12}$ and verified experimentally. ${ }^{3,4}$ The reason for this is that this convention permits one to preserve the relation between the external forces acting on the particle $F(r)$ and the Maxwell-Boltzmann probability distribution. More complex situations can be expected in absence of thermodynamic equilibrium, for example, in the presence of a temperature gradient. ${ }^{13}$

\section{EXPERIMENTAL SETUP AND METHODOLOGY}

For optical trapping we use dynamic computer-generated holograms displayed into a liquid crystal display (LCD). The method is based on lens and gratings algorithm. Briefly, the method enables the creation of multiple optical traps at arbitrary three-dimensional locations and a Spatial Light Modulator (SLM) permit updating the displayed holograms at video rates. Here, a collimated and expanded infrared $976 \mathrm{~nm}$ CW Nd:Yag laser beam from Alphalas is used as a source of highly coherent light. In the phase modulation stage, the wavefront 


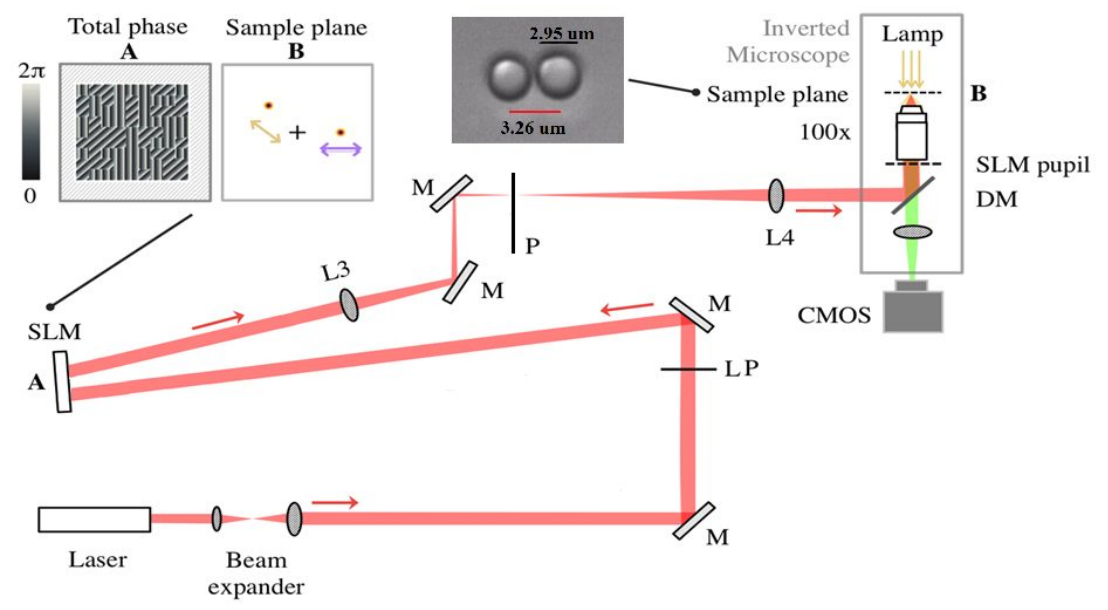

Figure 1: Schematic of the experimental setup. $\mathrm{LP}=$ linear polarizer. $\mathrm{P}=$ paddle, laser shutter. The lenses L3 and L4 form the image of the field in section B of the SLM in the output pupil of the microscope objective.

is modified by a Pluto NIR II-series SLM from Holoeye. The algorithm was programmed in Matlab language where an operative interface permit to create two independent optical traps in the focus region of an inverted objective lens 100x oil immersed from Edmund Optics.

Trapping power is typically in the range from 1 to $10 \mathrm{~mW}$ for each trap where two $2.95 \mathrm{um}$ PMMA modified acrylate particle from Kisker-Biothec are independently held. PMMA particles have weaker negative charge in contrast to standard polyestirene or silica beads, thus any electrostocatic or Van der Walls type effect at the surfaces are avoided. Sample was prepared diluting a highly concentrate solution of $10 \% \mathrm{w} / \mathrm{v}$ of PMMA particles in water. The final concentration of particles was $0.01 \% \mathrm{w} / \mathrm{v}$. Triton $\mathrm{x}-100$ was added to the sample with the aim to avoid them from sticking to glass. The final concentration of Triton X-100 was $0.8 \% \mathrm{v} / \mathrm{v}$ and the viscous coefficient was 2.1 (in relation to water).

White light illumination and camera permit simultaneous visualization, video acquisition and position register of the particles in real-time. Visualization and video acquisition were performed with a CMOS monochrome USB Camera from Thorlabs connected to a PC. The technology of the CMOS camera permit to select a small region of interest (ROI) in order to visualize only the particles of interest at high frame rate per seconds (fps) at the expense of pixels resolution, from $30 \mathrm{fps}$ at the maximal field of view of $1280 \mathrm{x} 1024$ pixels up to $100 \mathrm{fps}$ at $144 \times 108$ pixels ROI. The register method is based on particles position detection by threshold grayscale with sub-pixel accuracy, up to $80 \mathrm{~nm} /$ pixel. In order to perform statistics the experiment follows an automatic sequence engineered in Labview. Here, the laser beam can be switched on/off with a paddle connected to a motor-step actuator from Thorlabs. First, the sequence starts when the paddle is in on-position and the two particles are trapped. Then, the paddle is in off-position and the laser path is interrupted, here video acquisition start and particles diffuse for a few seconds. Video acquisition stops when the paddle is in off-position again and particles are trapped back to the original position. Immediately after, in between a delay time, video analysis and position register is executed. Finally, each video and its spatio-temporal data vector are stored in a buffer and the sequence repeats. Typically more than 2000 videos are acquired at an acquisition frequency of $100 \mathrm{fps}$ during an acquisition time of 4 seconds, which correspond to approximately 800.000 position data.

The optical tweezers setup is mounted on an air-suspended optical table from Thorlabs for achieving maximal isolation, while all possible noise sources such as power supplies and ventilators are placed away from the table. For details see Fig. 1.

\section{RESULTS}

Fig. 2 shows the experimental results. First, two PMMA neutral acrylate particles are held by two independent optical traps and placed in proximity as long the optical forces permit to avoid hopping. This is less than 300 
[nm] surface to surface, but taking care to avoid contact in order to minimize the effect of mechanical collision. Particles to coverglass distance is less than $5[\mu \mathrm{m}]$ in this example.
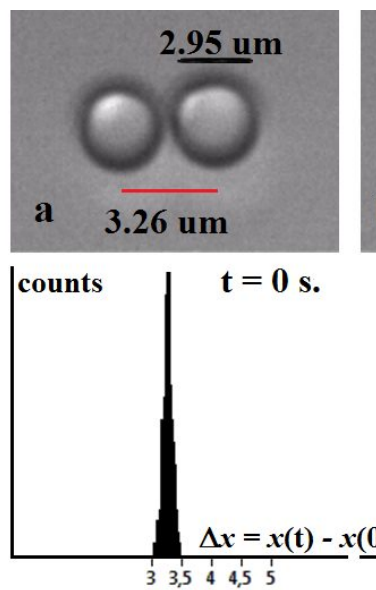
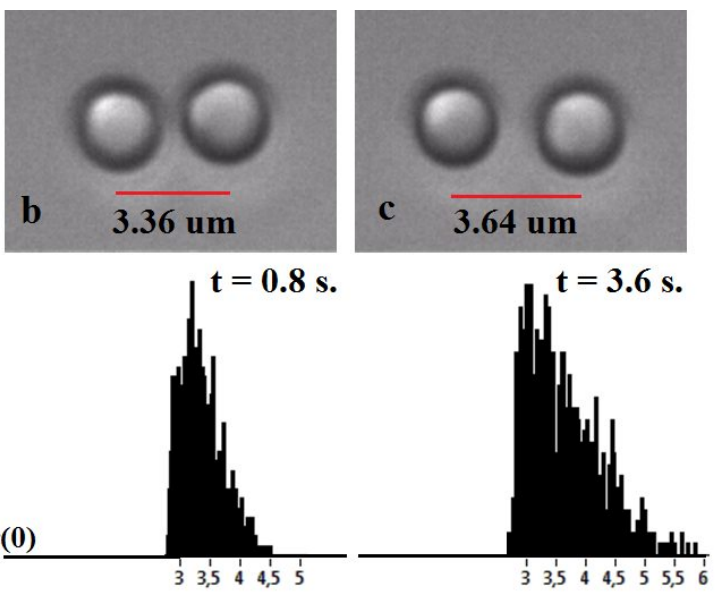

Figure 2: Three images sequences of a 4 sec. video register at $t_{0}=0, t_{1}=0.8$ and $t_{2}=3.60$ seconds respectively. Bottom: equation 5 .

In a standard 4 seconds-100 fps video acquisition the register initialize at the moment the traps are switched off and rapidly the particles diffuse. Here, $x(t)$ refers to the progressive separation between the centers of the beads. Thus, the surface to surface distance is easily calculated subtracting the radio of the particle. Three snapshots at $x(0)=3.26 \mu \mathrm{m}, x(0.8)=3.36 \mu \mathrm{m}$ and $x(3.6)=3.64 \mu \mathrm{m}$ clarified the evolution of the diffusion parameter as can be seen on the successive variances registers taken from the statics of the experiment.
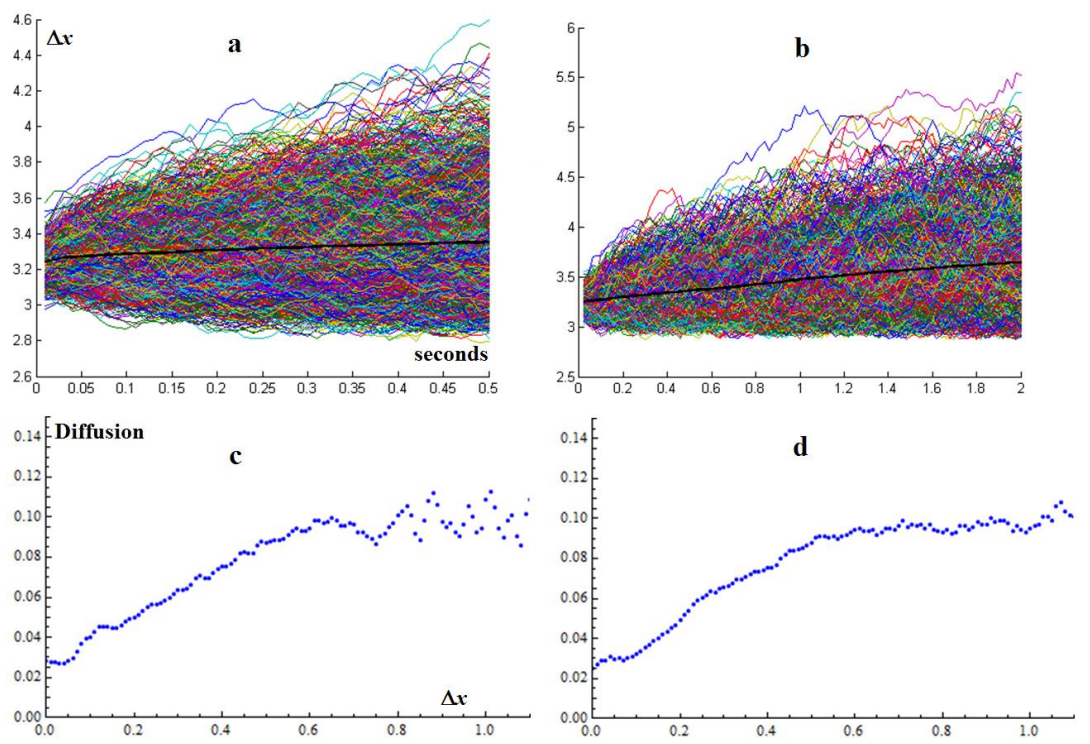

Figure 3: Up: 1000 trajectories plotted for acquisition times of $t=0.5$ seconds at $100 \mathrm{fps}$ (a) and 2 sec. at 50 fps(b). Bottom: Calculated diffusion, in units of $(\mu m)^{2} / s e c$.

Brownian trajectories for acquisition times of $t=0.5$ seconds and 2 sec. are plotted in fig. 3 for comparison. The particles to coverglass distance is less than $5[\mu \mathrm{m}]$. Greater separations must be analysed with 

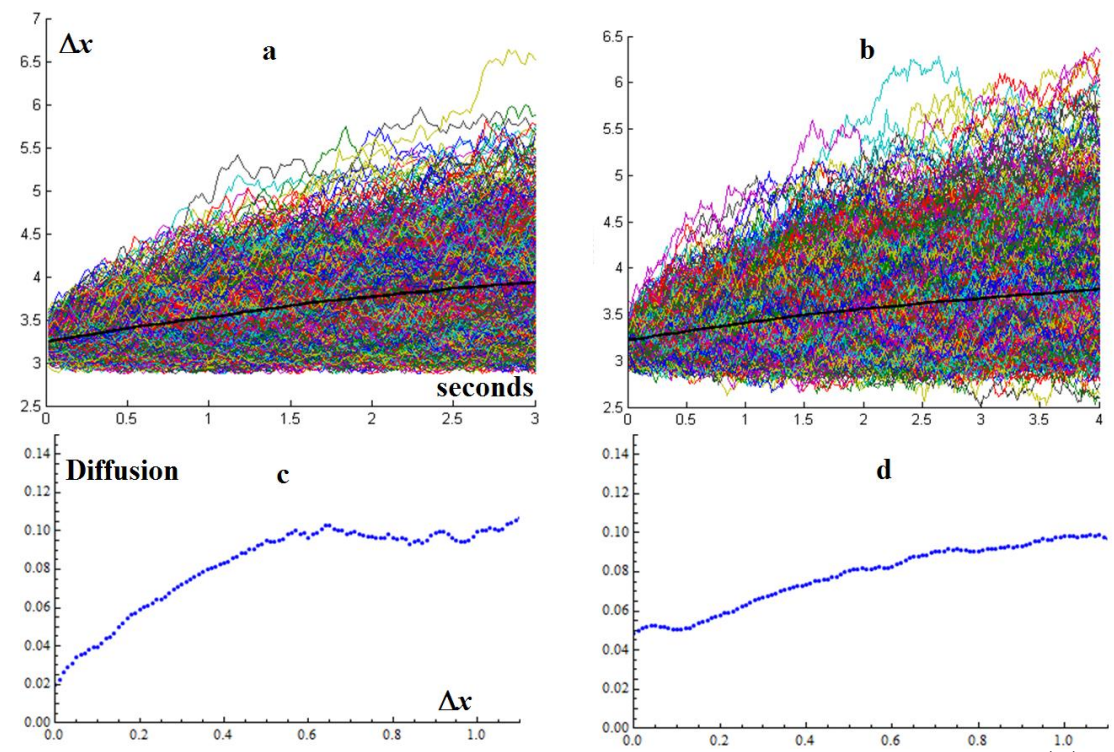

Figure 4: Up: 1000 trajectories plotted for acquisition times of $t=3$ seconds at $50 \mathrm{fps}$ (a) and 4 sec. (b) at 100 fps. Bottom: Calculated diffusion, in units of $[\mu \mathrm{m}]^{2} / \mathrm{sec}$.

care in a future work, because the estimated resolution in units of $\mathrm{nm} /$ pixel decrease by effects of defocusing due to gravity. Thus, the resolution must be calibrated dynamically in real-time in order to ensure data reliability.

Brownian trajectories for acquisition times of $t=3$ seconds and 4 sec. are plotted in fig. 4 for comparison. The particles to coverglass distance is less than $5[\mu \mathrm{m}]$ as in the previous figure.

\section{CONCLUSIONS}

It is a task to perform a real-time spatial control of diffusion gradients by setting dynamic wall geometries. This would allows to characterize Brownian trajectories of colloidal micro-particles diffusing in between a deformable channel. In a thought experiment, a real-time feedback control mechanism via holographic optical traps is engineered to adjust the position of the channel -multiple trapped particles- in response to information of the particle drift.

\section{ACKNOWLEDGEMENTS}

This work was partially supported from PIA-CONICYT PFB0824. J.P.Staforelli acknowledge support from FONDECYT 11110145. Ignacio Bordeu is supported by a CONICYT Master Scholarship.

\section{REFERENCES}

[1] Brenner, H., "The slow motion of a sphere through a viscous fluid towards a plane surface," Chem. Eng. Sci. 16, 242-251 (1961).

[2] Franosch, T., Grimm, M., Belushkin, M., Mor, F. M., Foffi, G., Forró, L., and Jeney, S., "Resonances arising from hydrodynamic memory in brownian motion," Nature 478(7367), 85-88 (2011).

[3] Volpe, G., Helden, L., Brettschneider, T., Wehr, J., and Bechinger, C., "Influence of noise on force measurements," Phys. Rev. Lett. 104(17), 170602 (2010).

[4] Brettschneider, T., Volpe, G., Helden, L., Wehr, J., and Bechinger, C., "Force measurement in the presence of brownian noise: Equilibrium-distribution method versus drift method," Phys. Rev. E 83(4), 041113 (2011). 
[5] Purcell, E. M., "Life at low reynolds number," Am. J. Phys 45(1), 3-11 (1977).

[6] Lançon, P., Batrouni, G., Lobry, L., and Ostrowsky, N., "Drift without flux: Brownian walker with a space-dependent diffusion coefficient," EPL (Europhysics Letters) 54(1), 28 (2001).

[7] Itō, K., "Stochastic integral," Proc. Imp. Acad. 20, 519-524 (1944).

[8] Stratonovich, R. L., "A new representation for stochastic integrals and equations," J. SIAM Control 4, 362-371 (1966).

[9] Klimontovich, Y., "Itô, stratonovich and kinetic forms of stochastic equations," Phys. A 163, 515-532 (1990).

[10] van Kampen, N. G., "Itô vs. Stratonovich," J. Stat. Phys. 24, 175-187 (1981).

[11] Clark, A. T., Lal, M., and Watson, G. M., "Dynamics of colloidal particles in the vicinity of an interacting surface," Faraday Discuss. Chem. Soc. 83, 179-191 (1987).

[12] Hottovy, S., Volpe, G., and Wehr, J., "Noise-induced drift in stochastic differential equations with arbitrary friction and diffusion in the Smoluchowski-Kramers limit," J. Stat. Phys. 146, 762-773 (2012).

[13] Hottovy, S., Volpe, G., and Wehr, J., "Thermophoresis of brownian particles driven by coloured noise," EPL (Europhysics Letters) 99(6), 60002 (2012). 